\title{
El futuro de las revistas contables colombianas: un debate polifónico
}

The Future of Colombian Accounting Journals: a Polyphonic Debate

\section{O futuro das revistas contábeis colombianas: um debate polifônico}

doi:10.11144/Javeriana.cc15-37.frcc

Hugo A. Macías

Compilador

Sorany Alzate, Jairo Alonso Bautista, Abel Cano Morales, Carlos Eduardo Castaño, Raquel Ceballos-Molano, Jhon Henry Cortés, Carlos Emilio García, Yuliana Gómez-Zapata, Hugo A. Macías, José Joaquín Ortiz-Bojacá, Olver Quijano-Valencia, Gloria Isabel Rodríguez, Gabriel Rueda-Delgado, Héctor José Sarmiento 
Como respuesta a la invitación que hizo el profesor Gabriel Rueda (2012), los profesores Mauricio Gómez-Villegas, Edison Fredy León y Hugo A. Macías han participado en un debate formal sobre el estado actual y el futuro de las revistas contables colombianas. La primera versión del debate fue publicada en diciembre de 2012 en la revista Investigación y Reflexión, mientras que las versiones dos y tres se publicaron en Cuadernos de Contabilidad, ambas en 2013. En esta oportunidad, nos hemos propuesto ampliar el debate, no solo a los editores de las revistas contables indexadas y sin indexar, sino a los editores de revistas de ciencias económicas y administrativas en las que han aparecido importantes discusiones contables.

Después de recibir la invitación personal extendida a cada uno de los editores para participar en el debate, en este texto se incluyen las apreciaciones de quienes hemos estado al frente de 14 revistas clave para la construcción colectiva de conocimiento contable en Colombia: Activos, Adversia, Colombian Accounting Journal, Contaduría Universidad de Antioquia, Cuadernos de Administración (Universidad del Valle), Cuadernos de Contabilidad, Criterio Libre, En-Contexto, Innovar, Lúmina, Porik An, Revista Colombiana de Contabilidad, Teuken-Bidikay y Visión Contable. Cada una de las contribuciones está ordenada alfabéticamente, por el apellido de los autores.

Para lograr la participación de este amplio grupo de editores y que el documento no quedara muy extenso, optamos por que cada uno escribiera unas 300 palabras y seguimos la estructura que se utilizó recientemente por un grupo de académicos europeos que discutieron el futuro de la corriente interpretativa en investigación contable (Ahrens, Becker, Burns, Chapman, Granlund, Habersam, Hansen, Khalifa, Malmi, Mennicken, Mikes, Panozzo, Piber, Quattrone \& Scheytt, 2008). Para no limitar temáticamente el aporte de cada editor, decidimos no seleccionar aspectos específicos de la discusión, sino que compartimos entre todos los textos ya publicados de versiones anteriores del debate (Gómez-Villegas, 2012, 2013; León, 2012; Macías, 2012, 2013a; Macías \& Moncada, 2011; Rueda, 2012, 2013). Esta cuarta versión fue coordinada por el profesor Hugo A. Macías, quien también hizo una nueva contribución; al final del texto presentamos los puntos de encuentro y desencuentro.

\section{Sorany Alzate ${ }^{1}$}

$\mathrm{Al}$ debate en el que varios pensadores contables han sumado sus aportes para consolidar un diagnóstico sobre las revistas contables colombianas, habría que agregar que el rezago que tenemos respecto a revistas internacionales es una consecuencia del déficit de inversión de los gobiernos en la investigación, lo cual no es un fenómeno exclusivo de la disciplina contable, sino que se replica en otras áreas del saber. A ese abandono y desinterés político por crear un clima científico en el país, pocos respondemos con convicciones férreas, al apostar por la investigación a pesar del sombrío panorama. Considero que no basta Colciencias, sobre todo cuando los proyectos que financia son los que

1 Editora de Adversia, revista virtual de estudiantes de contaduría pública, de la Universidad de Antioquia, Medellín. Correo electrónico: soranyalzate@gmail.com. 
le sirven a la corporación, por lo que la tendencia es a marginar los "proyectos no productivos" de las oportunidades de investigación.

Considerando lo anterior, queda sumar voluntades y esperanzas para que las revistas contables nacionales alcancen el status de las internacionales. Una posible ruta puede contemplar lo siguiente: 1) fortalecimiento metodológico en la investigación que finalmente derivará en artículos rigurosos; de esta forma, se les daría más visibilidad internacional a las reflexiones de pensadores colombianos, porque el empleo de metodologías formales los legitima y les confiere un aura de verdad ante la comunidad investigativa; debemos optar por un desarraigo de la investigación documental y la investigación reflexiva a priori ${ }^{2}$, y para trascenderlas se puede acudir a las metodologías que proponen las corrientes de investigación contable: la corriente ortodoxa, la corriente interpretativa y la corriente crítica (Alzate, 2014); 2) aprovechar los nuevos programas de maestría y los proyectos de doctorado para el mencionado fortalecimiento metodológico y para decantar trabajos de corte científico, de un rigor similar al de los artículos de las revistas internacionales; y 3) los comités editoriales de las revistas contables nacionales deben replantear el criterio que exige que los artículos sean inéditos; este criterio editorial cierra las puertas a los

2 El artículo El campo de la teoría contable, distinciones centrales para subrayar el papel de la Universidad de Antioquia en el contexto colombiano (Ospina, Castaño, Alzate \& Fernández, 2012) habla de esa preferencia por el uso de la investigación reflexiva a priori y de la investigación documental, al punto de que la metodología que propone la corriente hegemónica de investigación contable, a pesar de ser tan abundantemente trabajada en el ámbito internacional, tiene serias carencias en el plano nacional. trabajos presentados en eventos académicos, cuya publicación se limita a los libros de memorias; por el contrario, debería iniciarse una evaluación de los trabajos destacados para dejarlos a disposición de lectores diferentes a los asistentes a los eventos. Aunque en general algunas revistas están transitando hacia esa laxitud respecto de la exclusividad de los artículos, otras son estrictas en este criterio editorial.

Por último, quisiera plantear la necesidad de trascender el diagnóstico del tema de las revistas nacionales y pasar a la construcción de un plan para mejorar la calidad de los artículos publicados, lo cual permitirá que las revistas contables nacionales ganen status, no necesariamente frente a las revistas internacionales, sino que se piense una comunidad de revistas rigurosas, con dinámicas propias que, de ser necesario, abandone el referente internacional.

\section{Jairo Alonso Bautista ${ }^{3}$}

Sean fachadas de una casa sin terminar o proyectos de reconocimiento científico, las revistas académicas en Contabilidad son una fiebre de reciente aparición. El impulso se debe a los procesos de regulación de la calidad de la educación superior, el mayor progreso de la reflexión académica, que se atrevió con cierto ímpetu a "salir del closet" pues estaba acostumbrada a habitar una mayor conciencia institucional de la importancia de la investigación —que lamentablemente aún es marginal—y

3 Editor de la revista Activos, de la Universidad Santo Tomas, Bogotá, y de la Revista Colombiana de Contabilidad, publicada por la Asociación de Facultades de Contaduría Pública, ASFACOP.

Correo electrónico: jairobautista@usantotomas.edu.co. 
también, la afortunada cosecha de una generación de contadores y contadoras conscientes de su papel como intelectuales, muchos de ellos formados en el seno de procesos como el de la Federación Nacional de Estudiantes de Contaduría Pública, FENECOP, discípulos de maestros del legendario Centro Colombiano de Investigaciones Contables, C-CINCO, y lectores ávidos de los primeros textos de teoría contable traducidos al español.

Este contexto es el que ha permitido la emergencia de un número mayor de publicaciones en contabilidad con aspiraciones científicas; en ese espacio en consolidación, sobre las revistas pende la espada de Damocles: es necesario seguir los lineamientos de los grandes modelos de indexación y reconocimiento, propios de la ciencia normal, pero a la vez se cultivan artículos sobre un terreno que resulta estéril e insuficiente para alimentar todos los proyectos editoriales, y la calidad aún es muy dispar entre las diferentes publicaciones; el desarrollo depende fuertemente del interés de las instituciones y los aparatos logísticos que se disponen para el desarrollo de las revistas.

A pesar de ello, la opción es avanzar. Comparto con Fredy León la idea de que es necesario permitir que los procesos editoriales tomen caminos diferenciales y complementarios, que puedan crearse revistas especializadas, que los editores aportemos a la visibilización de todos los esfuerzos editoriales, que comencemos a trabajar en nuevas formas editoriales (convenios, números conjuntos y debates polifónicos como este), con el fin de soportar con mayor fuerza los cimientos de esta casa en construcción, y diseñar una arquitectura armoniosa que contribuya al desarrollo de la contabilidad en su sentido más universal, pero también en sus referentes locales más sentidos... en fin, estimular la creación de una ciencia propia.

\section{Abel Cano-Morales ${ }^{4}$}

La revista Colombian Accounting Journal se creó con el propósito de aportar a la superación de los límites y las fronteras del conocimiento contable, desde una publicación nacional que fuera conocida en el contexto internacional; no obstante, siendo esto lo que nos define, se ha convertido en una limitante, pues estamos conscientes de que los investigadores contables en Colombia son muy pocos y de que los artículos que se gestan en las principales instituciones académicas no son suficientes para atender el alto número de revistas contables; es decir, el número de investigadores o autores contables no está creciendo proporcionalmente al número de revistas.

Pero el problema, a mi juicio, surge de la educación que se está impartiendo en las aulas de clase, ya que los pocos profesores que se atreven a escribir, no son leídos, ni son citados, pues hay una serie de intrigas y descalificación de los trabajos hechos por unos pocos, a veces sin argumentos y en espacios no académicos. Además, debemos entender que por los diversos ambientes culturales en los cuales se desenvuelven los investigadores de la disciplina contable, tendemos a recrear comunidades de nuestro ambiente ancestral, redes de

4 Editor de la revista Colombian Accounting Journal, Universidad de Medellín, Medellín.

Correo electrónico: amcano@udem.edu.co. 
no más de tres a cinco personas, en las cuales se establecen relaciones jerárquicas y se asignan responsabilidades; tendemos a contar historias y a encontrar la oportunidad de expresarnos artísticamente, tenemos una orientación social.

Las revistas tienen un período de gestación de dos o tres años y es responsabilidad de los docentes, investigadores y académicos que las rodean enviarles sus trabajos inicialmente a estas; la endogamia en las primeras etapas es natural y necesaria. Por otro lado, es preciso tomar en cuenta la falta de confianza en sí mismos que tienen muchos investigadores contables, pues muy pocos confían en sus propias ideas, muy pocos van contra las adversidades y por ello sucumbimos ante la crítica y dejamos de expresar abiertamente nuestros deseos y nuestras ideas, no nos atrevemos a publicar lo que realmente pensamos de nuestra profesión o de nuestros actos profesionales.

\section{Carlos Eduardo Castaño- Ríos $^{5}$}

La situación actual de las revistas contables colombianas aparece quizás como un reflejo de las dificultades que se han producido para generar una cultura de comunidades investigativas en el campo del conocimiento contable. Sin duda alguna, el diagnóstico desalentador que se observa en el trabajo de Hugo A. Macías y Tatiana Moncada (2011) frente al posicionamiento de nuestras re-

5 Editor de la revista Contaduría Universidad de Antioquia, Medellín.

Correo electrónico: carloscontaduria@gmail.com. vistas en el ámbito internacional (desde la perspectiva de indicadores de inclusión en bases de datos denominadas de alto impacto), obedece a los mismos problemas que ha tenido la comunidad contable colombiana para integrarse en grupos de investigación consolidados. En este sentido, se debe notar que en la actualidad los niveles de formación de los profesores que hacemos parte de los diferentes programas de contaduría del país se vienen incrementando y que los grupos de investigación están mejorando sus dinámicas propias para aportar artículos que cumplan los requerimientos establecidos por las revistas contables (que se van ajustando a medida que se superan requisitos de bases de datos de indexación cada vez más rigurosas).

No obstante lo anterior, la discusión sobre las revistas debe pasar más por el tamiz de sus objetivos fundamentales. En este sentido, vale la pena retomar las palabras de la profesora María Eugenia Upegui (2012) en el editorial de la revista Contaduría Universidad de Antioquia, 60, con motivo de sus 30 años de existencia, en el cual se entiende que las principales acciones del proyecto editorial siempre han estado enfocadas hacia "la formación de estudiantes y profesores; la promoción de la investigación contable; el intercambio de conocimientos entre contadores nacionales y extranjeros; $y$ ha impulsado el desarrollo de la contabilidad en el país" (p. 11), elementos que deben primar en los procesos editoriales académicos y que seguramente cualificarán nuestra comunidad contable colombiana para ubicarla a futuro como referente de pensamiento de la comunidad internacional. 


\section{Raquel Ceballos-Molano, $\mathrm{PhD}^{6}$}

La revista Cuadernos de Administración, en los 49 números publicados entre 1976 y 2013, ha recogido una escasa producción académica contable, aunque la convocatoria incluye artículos, tanto para las escuelas de administración como las escuelas de contaduría.

No obstante, desde 1986, aparecen las primeras reflexiones acerca de las relaciones entre la epistemología y las disciplinas contables. En su No. 9, el artículo sobre el objeto de estudio de la contaduría Elementos para el análisis del currículo de Contaduría, de los profesores Jorge Burbano y Diego Delgadillo, es pionero. En el No. 11 aparecieron artículos como Unidad de análisis para el estudio del sistema de información contable (Burbano); El contador y la investigación (Ortiz-Sarmiento) y Prácticas contables en el Valle del Cauca (Henao \& Acuña).

Con la publicación de ediciones temáticas, el No. 17 de diciembre de 1990 fue dedicado a Contabilidad: teoría, investigación, práctica, un espacio que mostró la necesidad de la investigación crítica y la discusión de temas de actualidad o interés; así los artículos se relacionaron con los cambios sociopolíticos de los años 80 y 90 de apertura e internacionalización, con autores extranjeros: El papel de la teoría contable (R. L. Watts \& J. L. Zimmerman), Posibilidady utilidad de la teoría positiva de la contabilidad (R. D. Whitley).

6 Editora general de la revista Cuadernos de Administración (A2 Colciencias), Universidad del Valle, entre enero de 2011 y julio de 2013, Cali.

Correo electrónico: raquel.ceballos@correounivalle.edu.co.
Producto de un proceso de construcción colectiva de profesores de contaduría pública, en el No. 28 de 2002 aparecieron artículos caracterizados por la visibilidad de la crisis de confianza en las revelaciones contables-financieras de grandes empresas de países considerados líderes en la gestión de las empresas y los sistemas de información.

Los números entre 2007 y 2012 tampoco registran una tasa alta de artículos científicos contables, pero destacan temas como estados financieros (Ágreda), costeo integral (Ángel), sistemas de información (Solano), normas contables en empresas latinoamericanas (González), finanzas públicas (López \& Mesa), financiación de pyme (Rivera) y el proyecto educativo en contaduría pública (Rojas \& Ospina), con importantes aportes al estudio de la contaduría. Este panorama hará que la revista demande mayor participación de la comunidad contable nacional e internacional en sus próximas ediciones.

\section{Jhon Henry Cortés ${ }^{7}$}

En el ámbito académico global, las publicaciones en revistas toman cada vez una mayor importancia. Las revistas académicas hoy están tomando la forma de instituciones en las cuales es posible la creación, la socialización y la reproducción del conocimiento, pero no solo ello, las revistas académicas también constituyen escenarios vitales para definir, legitimar o proponer rutas y cursos de acción para diversos

7 Editor de la revista Visión Contable, Universidad Autónoma Latinoamericana, Medellín.

Correo electrónico: jhonhenrycortes@gmail.com. 
agentes: Estado, instituciones de educación superior, empresas, etc.

Hoy en el mundo entero evidenciamos una lucha - unas veces más encarnizada, otras veces no tanto- para definir cuáles son las mejores publicaciones, pero esta calidad no está dada por la cantidad de problemas sociales que contribuyan a resolver tales publicaciones, tampoco por generar mejores condiciones de vida para sus habitantes, menos por presentar propuestas alternativas al statu quo; esta calidad resulta definida por el cumplimiento de estándares, muchos de ellos sin un claro sentido social más allá del establecido por el mercado.

Solo existe lo que está publicado, lo demás no existe o, al menos, se pretende su inexistencia. Pero para que académicamente algo exista en el ámbito mundial, no solo se requiere que esté publicado, sino - lo más importante-dónde está publicado, y en qué idioma está publicado. Responder a estas preguntas necesariamente nos llevaría a reflexionar en torno a las actuales formas de colonización de una o varias culturas sobre otras, al desconocer, alienar, en ocasiones subyugar la posibilidad, la potencia, la esperanza del otro, de otra cultura, de otros contextos, de otros idiomas.

Esta dinámica global ha incidido en Colombia. En el contexto de las publicaciones académicas nacionales en contabilidad, han comenzado a proliferar diversas revistas, muchas de ellas orientadas fundamentalmente a apoyar los procesos de visibilización y acreditación institucional que, en ocasiones, hacen evidente la ausencia de un sentido social y disciplinar claro. Será necesario, entonces, desde las publicaciones contables en Colombia, reflexionar sobre el sentido de los distintos proyectos editoriales que se están construyendo.

\section{Carlos Emilio García-Duque, $\mathrm{PhD}^{8}$}

Del debate sobre el presente y futuro de las revistas de contaduría (Macías, 2013a) solo se pueden esperar beneficios. Primero, porque en la medida en que los editores de las revistas colombianas puedan compartir sus ideas al respecto, será posible comenzar un proceso de estandarización que permita mejorar paulatinamente la calidad de estas revistas. Segundo, porque además de identificar coincidencias en la evaluación de la calidad actual de las revistas y en el análisis prospectivo de lo que se puede lograr en el futuro inmediato, también podremos encontrar ideas novedosas que no habrían circulado tan rápidamente sin el debate y que pueden convertirse en guías para la acción. Tercero, porque a pesar de la homogeneidad temática, cada revista tiene sus propias dificultades y el conjunto total enfrentará en breve una nueva amenaza, de tal magnitud, que si no trabajamos conjuntamente para hacer algo al respecto, podríamos presenciar la desaparición de todas ellas en un relativo abrir y cerrar de ojos. Me refiero a las crecientes demandas que sobre la calidad impone el nuevo modelo de Publindex, demandas que si bien parecen razonables en el largo plazo, resultan excesivas para casi todas las revistas contables colombianas y para la mayoría de las revistas científicas que publican las

8 Editor de la revista Lúmina, Universidad de Manizales, Manizales.

Correo electrónico: cegarcia@unimanizales.edu.co. 
universidades del país. En efecto, la condición de estar integradas en la base de ISI es ya una condición difícil de cumplir para revistas que publican artículos fundamentalmente en castellano y que están dirigidas a un público local. Y si a esta exigencia se le añade la recomendación de apuntar a los primeros dos cuartiles de esta base de datos, la tarea se vuelve casi irrealizable.

Algunos de nuestros colegas editores se han referido a esta situación antes (Gómez-Villegas, 2012; León, 2012; Rueda, 2012), y han destacado los pros y contras de establecer como meta para una revista académica su inclusión en ISI. Como estoy convencido de que factores como el impacto y la visibilidad de los artículos son algo más que un adorno para el solaz de quienes supeditan el desarrollo de la academia y la cualificación de las revistas a los resultados de la cienciometría, quiero mencionar dos razones para postular como meta de una revista académica (si bien a mediano o largo plazo) su inclusión en esta base de datos. La primera es que la genuina circulación del conocimiento solo se logra cuando nuestras ideas consiguen generar alguna reacción entre los miembros calificados de nuestra comunidad académica y, como resulta evidente, esa academia no se puede circunscribir a nuestros estudiantes y colegas en el diminuto sistema universitario colombiano. La segunda es que las disciplinas (académicas o científicas) solo pueden progresar mediante el papel de la crítica y el escrutinio de las ideas y esa práctica es casi desconocida entre nosotros; de ahí que la única esperanza de participar en un debate crítico productivo pasa por las comunidades académicas robustas y desarrolladas cuyos trabajos se leen, comentan y critican precisamente en las revistas que hacen parte de esa base de datos.

\section{Yuliana Gómez-Zapata9}

Tal como lo plantearan los colegas que iniciaron esta discusión (Gómez-Villegas, 2012; León, 2012; Macías, 2012; Macías \& Moncada, 2011; Rueda, 2012), es absolutamente necesario que la comunidad académica contable colombiana revise y reconozca su contexto disciplinal y lectoescritural, pues aún no se logra avanzar significativamente en la construcción de una producción escrita que se reconozca y se debata en los ámbitos nacional e internacional.

Sin embargo, las dinámicas investigativas en contabilidad han venido en ascenso en los claustros universitarios (Gómez-Villegas, 2013) y la difícil tarea promovida por algunos docentes de pensar y repensar la disciplina que nos ocupa y la sociedad en la que vivimos, ha ido ganando fuerza y calando en el imaginario colectivizado de nuestros estudiantes. Empero, no se puede declinar en los esfuerzos particulares y colectivos que, como académicos y docentes, estamos llamados a emprender, pues incluso las acciones escriturales que se despliegan desde las universidades siguen siendo tímidas y, en muchos de los casos, carentes de rigor académico y profundidad investigativa.

Tanto los procesos de formación lectoescriturales como los de formación investi-

9 Directora editorial de la revista En-contexto, Tecnológico de Antioquia, Medellín.

Correo electrónico: ygomezza@tdea.edu.co. 
gativa (sin evitar insistir en mis intereses particulares como docente) constituyen focos de atención en el devenir de las publicaciones científicas en contabilidad en Colombia, en la medida en que docentes y estudiantes sigamos teniendo dificultades para expresar nuestras ideas de forma escrita, para construir nuevo conocimiento o por lo menos para recontextualizarlo. Por tanto, la tarea inaplazable es el reconocimiento e implementación de alternativas de enseñabilidad (Gómez-Zapata, 2012) académico-investigativas y lectoescriturales en el ejercicio de nuestra labor docente, que permitan el reconocimiento de la producción científica como elemento catalizador en la construcción de una comunidad académico-contable en Colombia.

\section{Hugo A. Macías ${ }^{10}$}

Escribir tiene sentido en la medida en que lo escrito y publicado se lea, en la medida en que la producción escrita sea consumida. En la academia contable colombiana, es necesario precisar si los lectores de las revistas son los estudiantes, los profesores, los administradores académicos, los profesionales o los investigadores. Si cada revista tiene más focalizado el auditorio al que va dirigida y sus autores lo tienen

10 Coordinador de la Maestría en Contabilidad, Universidad de Medellín, Medellín; excoordinador de la Sala de Ciencias Económicas y Administrativas de la Comisión Nacional de Aseguramiento de la Calidad de la Educación Superior, CONACES, Ministerio de Educación Nacional, Bogotá.

Correos electrónicos: hmacias@udem.edu.co y hmaciasc@eafit.edu.co claro, esto facilitaría no solo hacer seguimiento a su impacto, sino que permitiría diseñar estrategias de mejoramiento.

El amplio número de revistas contables colombianas permitiría focalizar la audiencia de cada una y definir una estructura diferenciada de los artículos, en términos de extensión, lenguaje utilizado, relación con la práctica, relación con la teoría, fuentes y métodos utilizados. En nuestra academia, tienen mayor presencia la investigación documental (Alzate, 2014) y los ensayos, y tenemos la costumbre de ignorar lo que han escrito nuestros colegas sobre el mismo tema (Macías, 2013a); entre nosotros prevalece además un "ambiente de no lectores" (Grajales, 2010), que es necesario mejorar, mediante estrategias deliberadas que se pueden liderar desde las revistas.

Para emprender acciones de mejoramiento, hay muchos caminos que han señalado las publicaciones posicionadas en los primeros lugares en la academia mundial, tanto orientadas hacia los profesionales, como orientadas al desarrollo de las disciplinas académicas (Macías, 2010); también están documentados los esfuerzos del profesor Anthony Hopwood en la creación de una revista contable alternativa con el mismo impacto que las principales revistas de corriente principal (Baker, 2011). La academia contable extranjera no solo gira alrededor de la corriente principal, la corriente crítica y la corriente interpretativa, sino que se está consolidando un grupo de policentrismos (Macías, 2013b), que permiten la adhesión a una academia contable abierta, creciente, integrada y plural. 


\section{José Joaquín Ortiz-Bojacá11}

El debate sobre el futuro de las revistas contables colombianas, llega en un momento oportuno, pues estamos presenciando un florecer de la divulgación y de la difusión del pensamiento contable en el país. Por ello, se hace acuciante que nos cuestionemos hacia dónde van nuestras revistas contables e interdisciplinares de las ciencias económicas, para potenciar el escenario natural y los incipientes recursos, que en buena hora se destinan a tan importante misión.

Tres aspectos se tornan relevantes para este esclarecimiento: 1. ¿Qué impacto social se busca en el desarrollo socioeconómico desde la construcción del conocimiento contable y qué papel debe jugar la difusión desde nuestras revistas en el campo disciplinar y profesional? O ¿solo debemos enfocarnos a la divulgación del pensamiento contable desarrollado por nuestros investigadores en la comunidad científica nacional y/o internacional? 2. ¿Cuál debe ser la mejor forma de inserción internacional desde lo local hacia lo global, o viceversa, para lograr un desarrollo científico adecuado a nuestras necesidades, reconociendo los desarrollos científicos de punta, sin importar su origen, sino su adecuación a los diferentes contextos en que se desarrollan? 3. ¿Cómo debemos impactar en el desarrollo curricular o académico a partir del conocimiento generado, difundido y/o divulgado desde nuestras revistas o las extranjeras?

11 Director científico de la revista Criterio Libre, Universidad Libre de Colombia, Bogotá.

Correo electrónico: jjoaquin.ortiz@gmail.com.
Pareciera que las respuestas no nos correspondieran a los editorialistas, pero muy al contrario considero que articular nuestra responsabilidad social con el diseño del desarrollo a largo plazo de nuestro quehacer de difusores y divulgadores del pensamiento contable desde las revistas científicas de nuestro país es ineludible y debe contextualizar nuestro plan de desarrollo que debe ser integracionista del cuerpo representativo de las revistas existentes y futuras, al cual nos debemos adaptar desde la perspectiva particular que se proponga cada una de ellas. Ello plantearía una pregunta final, que cierra el contexto de esta discusión: ¿Cuál es el modelo de evaluación de impactos (léase indexación), que debe medir la calidad de nuestras revistas, que se adecúe al plan de desarrollo que nos planteemos y que debe liderar el cuerpo de científicos y profesionales, desde la academia colombiana?

\section{Olver Quijano-Valencia, $\mathrm{PhD}^{12}$}

Imposible desconocer la importancia de las publicaciones y sus contribuciones a la dinamización del pensamiento en general y en nuestro caso, de la profesión y disciplina contable. Empero, desde hace algunas décadas se impone una suerte de excesivo formalismo, enciclopedismo, academicismo y seudorrigorismo tanto en la institucionalidad científica nacional (Colciencias) como en el ámbito universitario público y privado, todo esto en medio de una 'loca' carrera que va irremediablemente tras las "bue-

12 Director de la revista Porik An, Universidad del Cauca, Popayán. Correo electrónico: oquijano@unicauca.edu.co. 
nas" maneras de la razón imperial (Quijano, 2011). Se trata — como ya lo advirtió Ernesto Sábato-de estos "mitos del rigor" entendidos como "esa tendencia a conferir valor mágico a lo que está impreso" y en respuesta al principio 'publica o pereces' como forma hegemónica de la publicación y visibilización, incluso en medio de una sociedad predominantemente de tradición oral (Sábato, 1979).

Múltiples esfuerzos y proyectos editoriales deben justipreciar todos los parámetros del enciclopedismo para luego entrar en otras carreras 'locas' como la indexación (Publindex para Colombia) y los rankings internacionales, arquetipos de una parte del fenómeno paranoico que reduce el conocimiento al grafocentrismo e inobserva otras formas de producción y representación del conocimiento. De otro lado, se trata de esta moderna y contemporánea manera de ejercer una especie de endogamia propia de la sociedad de los expertos que actúa a modo de nuevos consejos inquisitoriales configurando el 'comunitarismo autista' en el que "la mayoría de sus fuentes de reconocimiento o de castigo provienen de la misma comunidad. La publicación de trabajos, por ejemplo, medida clave del éxito académico, depende exclusivamente del veredicto de unos 'referees' que son también miembros del mismo colectivo. No hay, en fin, ninguna necesidad real de comunicarse con el resto de la sociedad y de hecho podría ser, incluso, contraproducente. Todas las fuerzas que actúan son, pues, claramente centrípetas" (Brey, Innerarity \& Mayos, 2009, p. 32).

En medio de este panorama, hasta ahora incuestionado en el contexto de las publicaciones y esfuerzos editoriales en Colombia, también institucionalmente se insiste en la creación, fortalecimiento y movilización de publicaciones, unas inspiradas en excesivo academicismo y seudorrigorismo, y otras sin la pretensión paranoica y sin la militancia en el exacerbado entusiasmo productivo y competitivo que alimenta también algunas políticas institucionales (Colciencias) muchas veces en franca obstaculización a la fluidez de las diferentes formas de producción y circulación del conocimiento, en especial en tiempos de transformaciones profundas y de la deslocalización y descentramiento del conocimiento y de los saberes.

\section{Gloria Isabel Rodríguez- Lozano, PhD ${ }^{13}$}

Es innegable el impacto positivo que una "buena revista", académicamente hablando, tiene en el desarrollo de todas las disciplinas y saberes en cualquier parte del mundo; la academia contable colombiana no tiene por qué ser una excepción. Todo lo contrario, es urgente e imprescindible contar con revistas especializadas en temas contables; sobre todo cuando en nuestro país hay revistas muy importantes y con mucha trayectoria de tiempo atrás, pero en temas diferentes.

Me parece que el debate no debe ser en el sentido de qué tan importante es el rol que desempeñan las revistas especializadas en temas contables en nuestro país (o lo que sería aún peor: preguntarnos si desempeñan algún papel); puesto que en mi opinión es indiscutible que contar

13 Directora y editora general de la revista Innovar, Universidad Nacional de Colombia, Bogotá.

Correo electrónico: girodriguezl@unal.edu.co. 
con revistas especializadas trae, para todas las disciplinas, un motor de desarrollo que hace evolucionar la ciencia en sus múltiples aristas y que la academia — de manera natural-debe estar allí presente desempeñando un papel protagónico.

La cuestión importante, a mi juicio, es tener claro cuál es el direccionamiento al cual obedecen las revistas para que su impacto en la academia contable colombiana sea cada vez mayor y genere los desarrollos necesarios que propendan por una ciencia contable nacional cada vez más robusta y con mayor credibilidad ante la comunidad académica nacional e internacional. Cuestión nada fácil; no obstante lo que es claro para mí, es que no se debe permitir que estas revistas estén supeditadas a intereses grupales, sean ellos los que sean -incluso aquellos provenientes de la academia-; la preocupación debe estribar en mostrar de manera independiente los desarrollos provenientes de la academia. Además, dejar de lado la preocupación respecto a que la revista esté en lo que en nuestro medio denominamos "índices internacionales", para dar por hecho que el estar allí es prenda de "reconocimiento y buena calidad de la revista". Estarían en grave riesgo de caer en lo que he llamado ¿El fin justifica los medios? (Rodríguez, 2012).

\section{Gabriel Rueda-Delgado ${ }^{14}$}

La pregunta central en torno a un debate como este, respecto del futuro de las revistas aca-

14 Editor de la revista Cuadernos de Contabilidad, Pontificia Universidad Javeriana, Bogotá.

Correo electrónico: gabriel.rueda@javeriana.edu.co. démicas de contabilidad, que se ha abierto de manera insospechada para mí, no es la conveniencia (o inconveniencia) de jugar con las reglas del Sistema Publindex o si para que las revistas sean "importantes" debemos buscar que todas lleguen a ser publicaciones ISI en cuartiles superiores. La pregunta debería hacerse más bien respecto de ¿para qué revistas académicas?, ¿con qué alcance?, ¿con qué identidad?, ¿para quiénes?

Una revista contable puede pensarse para ser divulgada entre la comunidad de una Universidad, o para sus estudiantes de pre y posgrado y ello no implica que sea una publicación de menor importancia que otra que aspira a ser A1 en el Sistema Nacional de Ciencia y Tecnología.

Hemos tenido la oportunidad de disentir con varios colegas en el sentido de afirmar que publicación que no se ponga a circular en las "altas élites académicas" de ISI e incluso en idioma inglés, es, en su línea argumental un conocimiento perdido. Desde mi punto de vista, eso es darle al conocimiento de cualquier disciplina una visión muy utilitarista, que hace juego a unos intereses particulares que muchas veces se desconocen.

En mi experiencia como editor de la revista Cuadernos de Contabilidad, pienso que más significativo que el escalafón o la categoría, es el sentido de hacer revistas con calidad, evaluadas, que procuren cada vez más exigencia en lo que se publica. En mi caso, la identificación de pares académicos expertos en diversos temas contables e incluso identificar nuevas tendencias temáticas o nuevos abordajes o miradas a temas ya tratados es mucho más im- 
portante que el cumplimiento de indicadores para ser indexado.

Nuestro reto entonces no está en el escalafón; está en la construcción de comunidad académica contable activa, visible, dinámica, crítica, técnica, funcional, ligada a la regulación, analítica, interpretativa, cuantitativa o cualitativa y otros más, etc. Y construir comunidad implica diversidad, apertura, rigurosidad, proponer y, en últimas, ser para los demás. Ahí debería, a mi juicio, seguir discutiéndose el futuro de las revistas contables en Colombia.

\section{Héctor José Sarmiento ${ }^{15}$}

Habida cuenta de los válidos planteamientos suscritos por los participantes del debate en ediciones precedentes, debe concederse cierta licencia para disentir de algunos argumentos, aun a costa de causar disfonía en esta amena conversación. La intención es plantear como urgente la necesidad de hacer un alto en la carrera editorialista de las universidades colombianas para repensar la lógica que anima la dinámica de las publicaciones académicas, de manera que pueda reconstruirse una perspectiva latinoamericana del desarrollo de la sociedad y las disciplinas, mediante la investigación y las publicaciones especializadas.

No hay en este debate ni fuera de él, ninguna razón objetiva que obligue a asumir la perspectiva dominante de la investigación contable,

15 Director editorial de la Revista Latinoamericana de Investigación en Organizaciones, Ambiente y Sociedad Teuken Bidikay, Politécnico Colombiano Jaime Isaza Cadavid, Medellín, Universidad Nacional de la Patagonia San Juan Bosco, Argentina.

Correo electrónico: teuken-bidikay@elpoli.edu.co. centrada en procesos adheridos a la lógica de reproducción del capital financiero en los mercados bursátiles u otras dinámicas conexas, pues la pertinencia contextual es el criterio que prima en esa forma de pensar y eso ya está resuelto para el caso de las revistas líderes, que publican lo que el contexto organizacional anglosajón requiere para optimizar los factores determinantes de su desarrollo. Quizás el error que se ha cometido en Colombia y Latinoamérica, es que en el afán de emular la ruta de éxito de los grandes journals, se ha olvidado que lo que se debe publicar - con mayor prioridad y sin asomo de vergüenza- son los textos que propongan reflexiones y soluciones altamente pertinentes para los problemas y las condiciones del desarrollo socioeconómico de esta región del mundo.

No obstante, esta proposición disfónica requiere la compañía de ideas que, sin lesionar la calidad, puedan aportar fuerza cohesionadora, como la estrategia de redes de investigadores y la constante discusión de los textos, de forma que las revistas sean más un escenario de debate de la comunidad académica, que una plataforma de convenientes vindicaciones salariales.

\section{Puntos de encuentro y desencuentro en esta versión del debate}

Cada lector puede hacer su propia relación entre los textos presentados aquí y resaltar aquellos que le resulten más cercanos a su propia percepción del futuro de las revistas contables colombianas. La extensión del aporte hecho por cada uno de nosotros implicó dejar por fue- 
ra algunas apreciaciones, pero entendemos las virtudes de la síntesis y la posibilidad de participación que le abre a un grupo amplio de editores en un texto conciso. La estructura abierta y plural de este debate polifónico no impide identificar algunos puntos de encuentro y desencuentro entre los editores, al menos con carácter preliminar.

Es evidente que se plantean al menos dos posiciones diferenciales. Por un lado, se presentan las ventajas de adoptar un modelo de gestión de las revistas legitimado internacionalmente; mientras que, por el otro, se destacan los peligros de adoptar un modelo que no fue diseñado para nuestra región y que ignora aspectos centrales de nuestra cultura y nuestra sociedad. Uno de nosotros plantea que el debate solo puede traer beneficios, al abrir la posibilidad de construir una guía para la acción, que facilite el mejoramiento paulatino de las revistas; el conocimiento solo circula de manera genuina cuando genera alguna reacción de los integrantes de nuestra comunidad, cuando las ideas son controvertidas, lo cual es poco común en la academia contable colombiana (Carlos Emilio).

Reconocemos que las revistas académicas hoy son instituciones centrales para la construcción colectiva de conocimiento contable (Gloria y Jhon Henry), que la mayor formación de los profesores está conduciendo a que los artículos se ajusten cada vez más a criterios muy exigentes (Carlos Eduardo) y que estamos presenciando un "florecer" de la difusión del pensamiento contable (José Joaquín y Yuliana). Sin embargo, el número de investigadores y de sus productos está creciendo menos que el número de revistas (Abel) y el nuevo modelo de Publindex se está convirtiendo en una amenaza que puede hacer desaparecer este tipo de publicaciones si no trabajamos conjuntamente (Carlos Emilio); no es claro que ese sea el modelo de indexación que debemos seguir (José Joaquín). Es claro que las revistas contribuyen a la dinamización del pensamiento en general, de la profesión y de la disciplina (Olver) y que la producción científica es el elemento catalizador en la construcción de comunidad académico-contable (Yuliana), pero aún enfrentamos altos niveles de endogamia, que son naturales en las primeras etapas de las revistas (Abel) y necesitamos mejorar la visibilidad y el impacto de los artículos, que "son más que un adorno" (Carlos Emilio).

Se ha llegado a este nivel de madurez, pero al mismo tiempo de vulnerabilidad, por diferentes causas. Las revistas se han beneficiado en buena medida de los procesos de investigación desarrollados por Fenecop y por el C-Cinco (Jairo Alonso); una de las revistas de administración publicó importantes trabajos con bases conceptuales en la década de los ochenta, artículos orientados a la teoría contable en los años noventa y otros orientados a áreas más funcionales en la última década (Raquel). Sin embargo, la producción académica contable es escasa (Raquel), la calidad de los artículos es dispar entre las revistas (Jairo Alonso), falta rigor académico, profundidad investigativa y aún no se logra avanzar significativamente en una construcción escrita que se reconozca y sea debatida (Yuliana). Este diagnóstico desalentador se da porque la comunidad contable colombiana no se ha integrado en grupos de investigación consolidados (Carlos Eduardo), porque hay desinterés político en 
crear un clima científico en el país (Sorany), porque parte de nuestra academia está inmersa en un "ambiente de no lectores" (Hugo), porque las revistas están jóvenes y constituyen un espacio en consolidación (Jairo Alonso).

Varios de los integrantes de este debate polifónico asumieron una posición crítica frente a los criterios que Colciencias viene proponiendo como guía para el desarrollo de las revistas (Héctor José, Jhon Henry y Olver) ${ }^{16}$. Por un lado, se propuso explícitamente una "disfonía en esta amena conversación", al plantear que es necesario hacer un alto en la carrera editorialista siguiendo parámetros externos a la región, para construir una perspectiva latinoamericana de desarrollo de la sociedad y de las disciplinas; debe primar el criterio de pertinencia contextual; en Latinoamérica se ha cometido el error de emular la ruta de éxito de las grandes revistas internacionales, pero es necesario concentrarse en reflexiones y soluciones a los problemas de desarrollo de nuestra región (Héctor José).

Se planteó también que los estándares de calidad no tienen un claro sentido social, que ganar visibilidad en el ámbito mundial necesita reflexiones sobre las formas actuales de colonización y que la proliferación de revistas se ha dado en respuesta a procesos de acreditación, sin un claro sentido social y disciplinar; es necesario "reflexionar sobre el sentido de los distintos proyectos editoriales que se están construyendo" (Jhon Henry). También se planteó que estamos en una loca carrera tras las "buenas" maneras de la razón imperial; intentamos emular un "mito del rigor" escrito, en una sociedad en la que pre-

16 Otros participantes en el debate también asumen una posición crítica, pero esa no es su contribución central. domina la tradición oral; la interacción se da entre los autores y los evaluadores, pero no hay comunicación con el resto de la sociedad; los criterios externos obstaculizan la fluidez de diferentes formas de producción y circulación del conocimiento, en una época de saberes deslocalizados y descentrados (Olver).

Desde una posición diferente en el debate, se planteó que es urgente contar con revistas contables de alta calidad, como las que hay en Colombia de tiempo atrás, en áreas cercanas; el debate no puede ser por el sentido o por el papel de las revistas; estas hacen evolucionar la disciplina, juegan un papel protagónico; no se debe permitir que una revista esté supeditada a intereses grupales, la preocupación debe centrarse en los desarrollos provenientes de la academia (Gloria). En el mismo sentido, es necesario hacer esfuerzos para que las revistas contables nacionales alcancen estatus de internacionales (Sorany), la comunidad contable colombiana debe ser en el futuro un referente de pensamiento de la comunidad internacional (Carlos Eduardo), es necesario que nos integremos a la estructura policéntrica de la academia contable mundial (Hugo).

Otro punto de quiebre en el debate fue planteado por la integrante más joven de este grupo (Sorany), al señalar que es el momento de trascender el diagnóstico de las revistas y avanzar en la construcción de un plan. En este sentido, aparecen propuestas en varias direcciones. Por un lado, se conserva una de las posiciones asumidas en las versiones anteriores del debate, al proponer que se permita que las revistas avancen por "caminos diferenciales y complementarios", trabajar en "nuevas formas 
editoriales" para fortalecer los cimientos de la casa en construcción, diseñar una "arquitectura armoniosa" para crear una ciencia propia que relacione lo universal y lo local (Jairo Alonso); también se indicó que el único camino no es llegar a las bases internacionales o ascender en el sistema nacional de indexación, sino que una revista académica contable puede tener sentido para una universidad en particular o para los estudiantes (Gabriel). Por otra parte, se proponen esfuerzos sistemáticos para lograr fortalecimiento metodológico, aumentar la participación de las maestrías y los doctorados, así como reconsiderar la relación de las revistas con las ponencias presentadas en eventos (Sorany).

En lo relacionado con las propuestas, también se planteó que es necesario aclarar cuáles son los objetivos fundamentales de cada una de las revistas (Carlos Eduardo), convencer a los autores potenciales para que postulen sus documentos (Abel), aumentar la participación de la comunidad contable nacional e internacional en las revistas de administración (Raquel), definir en cada revista si su principal auditorio está integrado por estudiantes, profesionales o investigadores (Gabriel, Hugo y José Joaquín) y desarrollar estrategias deliberadas inspiradas en aquellas que han alcanzado mayor impacto en la academia contable mundial (Hugo). Una comunidad robusta lee sus trabajos, los comenta, los critica y esa interacción entre los académicos lleva a una revista al sistema ISI, para que allí esa actividad se intensifique (Carlos Emilio).

Hemos llegado al final de la versión cuatro de nuestro debate polifónico, con una participación amplia y plural, de la que emergen posturas que pueden conducir a reestructurar y cualificar las revistas contables colombianas. Ya hemos avanzado bastante en los diagnósticos y tal vez este espacio de encuentro aporte a la construcción colectiva de una academia contable en la que algunas revistas se especialicen en generar discusiones alrededor de problemas locales en el contexto regional, mientras que otras estén más interesadas en seguir lineamientos internacionales que permitan interactuar de manera deliberada con otros espacios académicos y encontrar espacio para lo local en un contexto internacional amplio. El profesor Gabriel Rueda nos invita en esta oportunidad a seguir discutiendo el futuro de las revistas contables colombianas, en el sentido de construir comunidad académica diversa, abierta y rigurosa.

\section{Referencias}

Ahrens, Thomas; Becker, Albrecht; Burns, John; Chapman, Christopher; Granlund, Markus; Habersam, Michael; Hansen, Allan; Khalifa, Rihab; Malmi, Teemu; Mennicken, Andrea; Mikes, Anette; Panozzo, Fabrizio; Piber, Martin; Quattrone, Paolo \& Scheytt, Tobias (2008). The Future of Interpretive Accounting Research: A Polyphonic Debate. Critical Perspectives on Accounting, 19, 840-866.

Alzate, Julieth Sorany (2014). Corrientes heterodoxas e investigación social-cualitativa, en respuesta a las limitaciones de la investigación contable ortodoxa. Teuken Bidikay, 5, en prensa.

Baker, Charles Richard (2011). A Genealogical History of Positivist and Critical Account- 
ing Research. Accounting History, 16 (2), 207-221.

Brey, Antoni; Innerarity, Daniel \& Mayos, Gonçal (2009). La sociedad de la ignorancia y otros ensayos. Barcelona: Infonomia.

Gómez-Villegas, Mauricio (2012). Revistas académicas en contabilidad: la búsqueda de sentido contextual. Revista Facultad de Ciencias Económicas: Investigación y Reflexión, XX (2), 9-13. Disponible en: http://www.sci.unal.edu.co/scielo. php?script $=$ sci_arttext $\&$ pid $=$ S0121$68052012000200001 \& \operatorname{lng}=\mathrm{es} \& n r m=$ iso

Gómez-Villegas, Mauricio (2013). El reconocimiento contextual y el aprendizaje de las publicaciones internacionales en contabilidad. Cuadernos de Contabilidad, 14 (34), 273-284. Disponible en: http://www. javeriana.edu.co/fcea/cuadernos_contab/ vol14_n_34/vol14_34_10c.pdf

Gómez-Zapata, Yuliana (2012). Pensar y renovar los modelos de educación contable: Una discusión pendiente. Teuken Bidikay, 3, 59-74.

Grajales, Jhonny (2010). La escritura de anteproyectos de investigación en un ambiente de no-lectores. El caso del curso de metodología de investigación contable en la Universidad del Valle, Sede Norte del Cauca. Contaduría Universidad de Antioquia, 57, 145159. Disponible en: http://aprendeenlinea. udea.edu.co/revistas/index.php/cont/article/ viewFile/15582/13525

León, Edison Fredy (2012). La comunidad contable y sus publicaciones: consideraciones modernas sobre su ser. Revista Facultad de Ciencias Económicas: Inves- tigación y Reflexión, XX (2), 13-17. Disponible en: http://www.sci.unal.edu.co/ scielo.php?script $=$ sci_arttext\&pid $=$ S012168052012000200001\&lng=es\&nrm=iso Macías, Hugo A. (2010). El campo de la investigación anglosajona en management: referente para la investigación colombiana en administración. Revista Ciencias Estratégicas, 18 (24), 167-182. Disponible en: http://www. redalyc.org/pdf/1513/151316944002.pdf

Macías, Hugo A. (2012). Las revistas académicas como plataforma para la construcción de conocimiento contable. Revista Facultad de Ciencias Económicas: Investigación y Reflexión, XX (2), 5-9. Disponible en: http:// www.umng.edu.co/web/revistas//revistavol-xx-no.-2

Macías, Hugo A. (2013a). Debate sobre el presente y el futuro de las revistas contables colombianas: acuerdos, desacuerdos y retos. Cuadernos de Contabilidad, 14 (34), 265271. Disponible en: http://www.javeriana. edu.co/fcea/cuadernos_contab/vol14_n_34/ vol14_34_10b.pdf

Macías, Hugo A. (2013b). Internacionalización de las revistas contables: un aporte al debate polifónico. Cuadernos de Contabilidad, 14 (35), 785-794. Disponible en: http://www. javeriana.edu.co/fcea/cuadernos_contab/ vol14_n_35/vol14_35_16.pdf

Macías, Hugo A. \& Moncada, Tatiana (2011). El campo científico de la contabilidad. Panorama internacional de las revistas. Lúmina, 12, 260-278. Disponible en: http://www. umanizales.edu.co/publicaciones/campos/ economicas/lumina/recursos/12/3_campo_ cientifico.pdf 
Ospina, Carlos Mario; Castaño, Carlos Eduardo; Alzate, Julieth Sorany \& Fernández, Juan Carlos (2012). El campo de la teoría contable. Distinciones centrales para subrayar el papel de la Universidad de Antioquia en el contexto colombiano. En Marco Antonio Machado (ed.). Huellas y devenir contable. Construyendo las rutas del pensamiento contable, 222-275. Medellín: Universidad de Antioquia.

Quijano, Olver Bolívar (2011). Eufemismos. Cinismo y sugestión en la actual ampliación del campo de batalla. Popayán: Universidad del Cauca.

Rodríguez, Gloria I. (2012). Editorial: ¿El fin justifica los medios? Innovar, 22 (45), 3. Disponible en: http://www.redalyc.org/ pdf/818/81824864001.pdf

Rueda, Gabriel (2012). El sentido de buscar que Cuadernos de Contabilidad sea en el futuro una revista incluida en ISI. Cuadernos de Contabilidad, 13 (32), 10-14. Disponible en: http://www.javeriana.edu.co/fcea/cuadernos_contab/vol13_n_32/vol13_32_0_Editorial.pdf

Rueda, Gabriel (2013). Debate sobre el significado de la calidad en las revistas académicas contables del país. Un debate que debe extenderse. Cuadernos de Contabilidad, 14 (34), 263-264. Disponible en: http://www. javeriana.edu.co/fcea/cuadernos_contab/ vol14_n_34/vol14_34_10.pdf

Sábato, Ernesto (1979). Sobre algunos males de la educación. En Ernesto Sábato. Apologías y rechazos. Barcelona: Seix Barral.

Upegui, María Eugenia (2012). Editorial. Contaduría Universidad de Antioquia, 60, 11-14.

- Fecha de recepción: 25 de septiembre de 2013

- Fecha de aceptación: 25 de marzo de 2014

\section{Para citar este artículo}

Alzate, Sorany; Bautista, Jairo Alonso; Morales, Cano-Morales, Abel; Castaño, Carlos Eduardo; Ceballos-Molano, Raquel; Cortés, Jhon Henry; García, Carlos Emilio; Gómez-Zapata, Yuliana; Macías, Hugo A.; Ortiz-Bojacá, José Joaquín; Quijano-Valencia, Olver; Rodríguez, Gloria Isabel; Rueda-Delgado, Gabriel \& Sarmiento, Héctor José (2014). El futuro de las revistas contables colombianas: un debate polifónico. Cuadernos de Contabilidad, 15 (37), 261-278. 